\title{
An improved deconvolution algorithm using B-splines for well-test data analysis in petroleum engineering
}

\author{
Wenchao Liu ${ }^{\mathrm{a}}$, Yuewu Liu ${ }^{\mathrm{a}, *}$, Guofeng Han ${ }^{\mathrm{a}}$, Jianye Zhang ${ }^{\mathrm{b}}$, Yizhao Wan ${ }^{\mathrm{a}}$ \\ a Institute of Mechanics, Chinese Academy of Sciences, Beijing 100190, China \\ ${ }^{\mathrm{b}}$ Research Institute of Exploration and Development, Tarim Oilfield Company, Petro China, Korla, Xinjiang 841000, China
}

\section{A R T I C L E I N F O}

\section{Keywords:}

Deconvolution algorithm

Well testing

Duhamel principle

Least square method

\begin{abstract}
A B S T R A C T
Ilk et al.'s deconvolution algorithm using B-splines involves the Laplace transformation of the convolution equation with respect to production rate and wellbore pressure based on Duhamel principle. However, for common cases, the production rate function has "discontinuity" with respect to production time; it does not satisfy the precondition that the function to be transformed by Laplace transformation should be continuous. This inherent defect may directly cause enormous amount of computational time or even the failure of the numerical Laplace inversion in the deconvolution process. Based on these concerns, a fundamentally improved deconvolution algorithm using B-splines is presented here. In the convolution equation, the wellbore pressure derivative corresponding to constant unit production rate as the target of deconvolution is still represented by weighted summation of second-order B-splines; however, the computation process of the deconvolution is kept in the level of integral in the real time space instead of the Laplace space, for the reason that there will be no continuity requirement for the production rate function in the application of Duhamel principle for the deconvolution computation problem. According to the real production rate history, a technique of piecewise analytical integration is adopted for obtaining the elements of sensitivity matrix of a linear system with respect to weight coefficients; the linear system is generated by substituting the measured wellbore pressure data and corresponding variable production rate data into the convolution equation containing B-splines. The proposed direct analytical solution method of the integration for calculating the elements of the sensitivity matrix can not only guarantee the success of the deconvolution computation, but also can largely enhance the deconvolution computation speed. Moreover, in order to further improve the computation speed, a binary search method is also applied to find which production segments (with constant production rate) the measured wellbore pressure data points locate at in the deconvolution computation process. Another linear system with respect to weight coefficients for the regularization from Ilk et al.'s deconvolution algorithm is appended in order to overcome the effect of data errors. The two linear systems are combined together as an over-determined linear system, which can be solved by the least square method. Eventually, the reconstructed wellbore pressure and its derivative by $\mathrm{B}$-splines corresponding to the constant unit production rate can be obtained.

Numerical experimental tests demonstrate that the improved deconvolution algorithm exhibits good accuracy, computation speed and stability of data error tolerance. And the statement on how to perform the regularization when data error exists is also made in order to deconvolve the correct wellbore pressure derivative. The improved deconvolution algorithm is also applied into an actual field example. It is found that the deconvolution results by the improved deconvolution algorithm have good agreement with the ones by Von Schroeter et al.'s deconvolution algorithm and by Levitan et al.'s deconvolution algorithm as a whole; and the feature of typical log-log curves of the wellbore pressure drop and the wellbore pressure derivative corresponding to the improved algorithm is very close to the one of typical log-log curves calculated directly from the wellbore pressure data in the well shut-in period. In addition, through many numerical experimental tests, it is also concluded that as the quantity of data largely increases, the improved Ilk et al.'s deconvolution algorithm exhibits the big advantage in fast computational speed over von Schroeter et al.'s algorithm and Levitan et al.'s algorithm.
\end{abstract}

\footnotetext{
* Corresponding author.

E-mail address: liuyuewulxs@126.com (Y. Liu).
} 


\section{Introduction}

The production rate from the wellbore in the reservoirs can't keep constant all the time during the process of well testing. Therefore, for the well testing data, the measured wellbore pressure actually corresponds to the variable production rate at the whole time. However, it is well known that the reservoir models for the well-test analysis commonly have the inner boundary conditions with constant production rate. In order to conquer the inconsistency in reality, a pressure build up testing at the well shut-in period has been widely applied in reservoir engineering. Nevertheless, the investigation radius is short just for the shut-in period although the well test interpretation for the pressure buildup data can help to offer reliable results due to the less production rate measurement uncertainties. In order to make use of the well testing data corresponding to variable production rate at the whole production time, the deconvolution is introduced by researchers.

In a linear system as Darcy's flow, according to the Duhamel principle (Ilk, 2005; Vaferi and Eslamloueyan, 2015), the wellbore pressure under variable production rate can be obtained through a convolution, as follows:

$p_{\text {ini }}-p=\int_{0}^{t} q(t-\tau) p_{u}^{\prime}(\tau) \mathrm{d} \tau$

where $t$ is the time; $\tau$ is a variable for the integral; $q$ is the variable production rate; $p$ is the wellbore pressure corresponding to variable production rate; $p_{u}$ is the wellbore pressure drop corresponding to the constant unit production rate; $p_{\text {ini }}$ is the initial formation pressure. The purpose of the deconvolution process is to obtain the pressure response $p_{u}$ corresponding to the constant unit production rate with a duration equal to the whole production time, when the data of variable production rate $q$ and the corresponding wellbore pressure $p$ during the whole production time are both given.

The related deconvolution algorithms have been studied for more than 40 years. The main difficulty is that the deconvolution algorithm is very sensitive to the data error (from the measured wellbore pressure and production rate), which shows an inherent property of instability (Onur et al., 2006; Çınar et al., 2006). As far as we know, only three representative deconvolution algorithms with good stability are developed by von Schroeter et al. (2002, 2004), Levitan (2005); Levitan et al. (2006) and Ilk (2005), Ilk et al. (2005), respectively. They offer necessary stability to make the deconvolution as a viable tool for well-test analysis (Onur et al., 2006; Çınar et al., 2006); wherein just the two representative deconvolution algorithms by von Schroeter et al. (2002, 2004) and Levitan (2005); Levitan et al. (2006) have been implemented into Saphir as the pressure transient analysis module of KAPPA software due to their well performance. Von Schroeter et al.'s deconvolution algorithm and Levitan et al.'s deconvolution algorithm are both based on the same concept of minimizing a nonlinear weighted least-square objective function, involving the sum of three mismatch terms of pressure, rate and curvature, for reconstructing the deconvolved pressure drop and its logarithmic derivative; their difference mainly lies in the aspects of model assumption and specific definition of objective functions. In von Schroeter et al.'s deconvolution algorithm, it is assumed that a wellbore-storage unit-slope trend of typical curves of transient wellbore pressure is satisfied at or before the first node (Onur et al., 2006). However, in practice the wellbore-storage unit-slope trend is rare at the start of a transient wellbore pressure typical curve; and thus von Schroeter et al.'s deconvolution algorithm can not reconstruct the logarithmic pressure derivative corresponding to the constant unit production rate for the first log-cycle (Onur et al., 2006). In Levitan et al.'s deconvolution algorithm, the restriction assumption is removed by assuming that the time corresponding to the first node is sufficiently small. For the application of this kind of deconvolution algorithms base on optimization (minimization), Gringarten (2010) has given some recommendations on how to perform the deconvolution and verify the deconvolution results. It does provide great encouragement for the engineers to use deconvolution confidently as one part of the well test analysis process. At present, von Schroeter et al.'s deconvolution algorithm or Levitan et al.'s deconvolution algorithm has been further developed and extended. For example, based on a weighted Euclidean norm, Pimonov et al. (2009) improved the objective functions proposed by von Schroeter et al. and Levitan et al. The weights can be assigned to individual pressure and rate measurement points, and different error estimates are defined for different sections of data, which are very useful to mitigate of the effects of data errors. Cumming et al. (2013) extended the single-well deconvolution algorithm by von Schroeter et al. to the multi-well deconvolution algorithm by considering the interference effects among the wells. The feasibility of the extended deconvolution algorithm is also demonstrated by a synthetic example. Ilk et al.'s deconvolution algorithm (Ilk, 2005; Ilk et al., 2005) is another different method. It is formulated by using 2-order B-splines for representing the derivative of the wellbore pressure $p_{u}^{\prime}$ corresponding to the constant unit production rate, and requires numerical inversion of the Laplace transform; and a regularization method is implemented for handling the high levels of data errors. It is worth to mention that although these aforementioned deconvolution algorithms are only valid for linear systems i. e. reservoir models of Darcy's flow with slightly compressible fluid when Duhamel principle holds, they can be also applied to nonlinear systems through the linearized relationship between the defined pseudopressure and the production rate in the cases of gas or multiphase flow (Kim et al., 2015).

Onur et al. (2006) have presented an investigation of these three deconvolution algorithms, and discussed the specific features associated with the use of each algorithm. For the two deconvolution algorithms by von Schroeter et al. (2002, 2004) and Levitan (2005); Levitan et al. (2006), it has to be assumed that the variable production rate function should be given in a stepwise manner. In contrast, Ilk et al.'s deconvolution algorithm (Ilk, 2005; Ilk et al., 2005) allows for a general production rate function but with the restriction that the function must be transformed into Laplace space as an analytical formula. In view of this, Ilk et al.'s deconvolution algorithm is applicable for more cases. However, there still exist two problems in Ilk et al.'s deconvolution algorithm, as follows:

First, due to the fact that the 2-order B-spline functions are defined piecewise (first-order derivable at the knots of B-spline functions), Laplace inverse transformation would contain "discontinuity" in its higher derivatives. Moreover, whenever the production rate undergoes an abrupt change, the required condition for a successful numerical Laplace inversion is not satisfied (the function transformed by Laplace transformation should be continuous). In particular, the inverse Laplace transformation can fail at the discontinuities when computing the elements of the sensitivity matrix; therefore, the success or failure of Ilk et al.'s deconvolution algorithm depends primarily on the numerical Laplace transform inversion (Ilk, 2005; Ilk et al., 2005). In order to keep safe numerical inversion, the Gaver-Wynn-Rho algorithm (Ilk, 2005; Ilk et al., 2005) is used to improve numerical Laplace inversion at the discontinuity points, but higher values of precision (at least 64 digits or over) have to be used for accuracy. However, on one hand, the failure in the inverse Laplace transformation may still happen at the discontinuities despite using very high precision (Ilk, 2005; Ilk et al., 2005); on the other hand, even if the accurate results are obtained with using very high precision, this takes enormous amount of computational time which makes it impossible to apply for practical purposes (Ilk, 2005; Ilk et al., 2005). Although Ilk (2005) and Ilk et al. (2005) try to overcome these issues by modifying the rate approximation by dividing the production rate history into any number of segments, and use the type of function whose Laplace transform exists for approximating the rate within each segment, the effect is not good: As the number of segments increases, the computational time will increase significantly (Ilk, 2005; Ilk et al., 2005).

Second, it has been known that Ilk et al.'s deconvolution algorithm 
requires accurately fitting the discrete production rate data through continuous functions for every segment. What's more, it is also required that these continuous functions can be transformed into Laplace space as an analytical formula (Ilk, 2005; Ilk et al., 2005). The data fitting job by transformable functions for every segment introduces big inconvenience in the application of Ilk et al.'s deconvolution algorithm.

In fact, from the point of view of mathematics, the two aforementioned problems in Ilk et al.'s deconvolution algorithm can be attributed to the Laplace transformation of the convolution equation (i. e., Eq. (1)). In the convolution equation, there is no continuity requirement for the variable production rate function $q$ and the derivative of wellbore pressure function $p_{u}^{\prime}$ in the integral. In other words, even if the two functions have finite "discontinuity" points with respect to production time, the integral can still hold. However, it is known that the function to be transformed by Laplace transformation should be continuous; therefore, when Eq. (1) is transformed by Laplace transformation, the continuity requirement for $q$ and $p_{u}^{\prime}$ will be higher: They have to be continuous. Then if the functions in the convolution equation have large discontinuity, which is common in reality, Eq. (1) cannot be transformed correctly by Laplace transformation; and the subsequent numerical Laplace inversion will become very difficult in the deconvolution computation process.

Based on these concerns, Ilk et al.'s deconvolution algorithm is improved fundamentally here. The whole computation process of the deconvolution will be implemented in the level of integral, which has lower continuity requirement for functions in the convolution equation; Laplace transform is not involved. It avoids the unsuccessful Laplace transformation and its inverse in Ilk et al.'s deconvolution algorithm when the function of production rate is commonly discontinuous. The key computation techniques include: according to the real production rate history, a technique of piecewise analytical integration is adopted for obtaining the elements of sensitivity matrix of a linear system (Ilk, 2005; Ilk et al., 2005) with respect to weight coefficients in the deconvolution computation process. The piecewise analytical integration technique also conquers the difficulty in the time-consuming numerical computation of the integral for the case of production rate history with large discontinuity, and can largely improve the deconvolution computation speed. Furthermore, obviously, because the Laplace transformation of the convolution equation is avoided, the treatment for fitting the production rate data through transformable functions for every segment will be not involved in our improved deconvolution algorithm.

When significant errors exist in the data, the regularization method from Ilk et al.'s deconvolution algorithm (Ilk, 2005; Ilk et al., 2005) is still incorporated. The effect of data error on the deconvolution computation can be largely eliminated through selecting the logarithmic distribution of B-spline knots and tuning the regularization parameter simultaneously.

\section{Improvement of Ilk et al.'s deconvolution algorithm}

A spline function can be represented by the linear combination of B-spline functions. When the knots are set, the generation of B-splines is easy due to their intrinsic recurrence relation. The knots are distributed logarithmically, as follows (Ilk, 2005; Ilk et al., 2005):

$t_{i}=b^{i}, b>1 \quad i=0, \pm 1, \pm 2, \ldots$

where $b$ is the base; $t_{i}$ is the knot; $i$ is the index for the knots. In order to reveal the characteristic reservoir behavior, the number of knots should be on the order of at least 2-6 knots per log cycle, as Ilk et al. suggested (Ilk, 2005; Ilk et al., 2005).

When the value of the base $b$ is determined, the $i$-th zero-order Bsplines are defined as follows (Ilk, 2005; Ilk et al., 2005):
$B_{i}^{0}= \begin{cases}1 & t_{i}<t<t_{i+1} \\ 0 & \text { otherwise }\end{cases}$

Then higher order B-splines are generated recursively, as follows (Ilk, 2005; Ilk et al., 2005):

$B_{i}^{k}(t)=\left[\frac{t-t_{i}}{t_{i+k}-t_{i}}\right] B_{i}^{k-1}(t)+\left[\frac{t_{i+k+1}-t}{t_{i+k+1}-t_{i+1}}\right] B_{i+1}^{k-1}(t)$

where $k$ is the order of $\mathrm{B}$-splines.

Here, the derivative of wellbore pressure function $p_{u}^{\prime}$ corresponding to the constant unit production rate is also considered as a spline function, which can be represented by a weighted summation of 2order B-splines (Ilk, 2005; Ilk et al., 2005):

$p_{u}^{\prime}(t-\tau)=\sum_{i=1}^{u} c_{i} B_{i}^{2}(t-\tau)$

where $c_{i}$ is the undetermined weight coefficient; $u$ is the number of undetermined coefficients.

The objective is to determine the values of $c_{i}$ by using the measured wellbore pressure and corresponding production rate data.

According to the property of convolution, Eq. (1) can be rewritten as follows:

$p_{\text {ini }}-p=\int_{0}^{t} p_{u}^{\prime}(\tau) q(t-\tau) \mathrm{d} \tau=\int_{0}^{t} p_{u}^{\prime}(t-\tau) q(\tau) \mathrm{d} \tau$

Substituting Eq. (5) into Eq. (6) yields:

$$
\begin{aligned}
p_{\text {ini }}-p=\int_{0}^{t} p_{u}^{\prime}(t- & \tau) q(\tau) \mathrm{d} \tau \\
& =\int_{0}^{t}\left[\sum_{i=1}^{u} c_{i} B_{i}^{2}(t-\tau)\right] q(\tau) \mathrm{d} \tau \\
& =\sum_{i=1}^{u} c_{i} \int_{0}^{t} B_{i}^{2}(t-\tau) q(\tau) \mathrm{d} \tau
\end{aligned}
$$

It is assumed that the number of production segments is $m$ with different constant production rate $q_{j}, j=0,1, \ldots, m-1$. The time domain corresponding to the production segment with the production rate $q_{j}$ is $\left[T_{j}, T_{j+1}\right)$, which covers $n_{j}$ measured wellbore pressure data $\left(T_{k}^{j}, p_{k}^{j}\right)$, $k=0, \ldots, n_{j}-1$; where $T_{0}^{j}=T_{j}, T_{n_{j}}^{j}=T_{j+1}$.

Then the integral $\int_{0}^{t} B_{i}^{2}(t-\tau) q(\tau) \mathrm{d} \tau$ in Eq. (7) can be split into several independent integrals according to the production segments with different production rates (see Fig. 1), as follows:

$\int_{0}^{t} B_{i}^{2}(t-\tau) q(\tau) \mathrm{d} \tau$

$=\int_{0}^{T_{1}} B_{i}^{2}(t-\tau) q_{0}(\tau) \mathrm{d} \tau+\int_{T_{1}}^{T_{2}} B_{i}^{2}(t-\tau) q_{1}(\tau) \mathrm{d} \tau+$

$\int_{T_{2}}^{T_{3}} B_{i}^{2}(t-\tau) q_{2}(\tau) \mathrm{d} \tau+\ldots \ldots+\int_{T_{l}}^{t} B_{i}^{2}(t-\tau) q_{l}(\tau) \mathrm{d} \tau$

where $t \in\left[T_{l}, \quad T_{l+1}\right)$.

In Eq. (8), the discontinuity of the piecewise constant production rate function $q(\tau)$ at $T_{l}$ does not affect the integration result. It is very convenient for computing the elements of sensitivity matrix (Ilk, 2005; Ilk et al., 2005) for the deconvolution problem. It is also found through numerical tests that if the technique of integral splitting is not taken, the deconvolution results will be distorted seriously. The split integrals in Eq. (8) can be solved analytically, as follows:

$\int_{T_{i}}^{T_{i+1}} B_{i}^{2}(t-\tau) q_{i}(\tau) \mathrm{d} \tau=q_{i} \cdot \int_{0}^{t-T_{i}} B_{i}^{2}(\tau) \mathrm{d} \tau-q_{i} \cdot \int_{0}^{t-T_{i+1}} B_{i}^{2}(\tau) \mathrm{d} \tau$

$\int_{T_{l}}^{t} B_{i}^{2}(t-\tau) q_{l}(\tau) \mathrm{d} \tau=q_{l} \cdot \int_{0}^{t-T_{l}} B_{i}^{2}(\tau) \mathrm{d} \tau$

where the integral of $B_{i}^{2}(t)$ in the above equations can be analytically formulated from the integrals of the piecewise polynomials of Bsplines.

It should be noted that when $q_{i}$ is a general production rate function with respect to $t$, Eqs. (9) and (10) will not hold. However, this problem can be solved by further approximating the production 


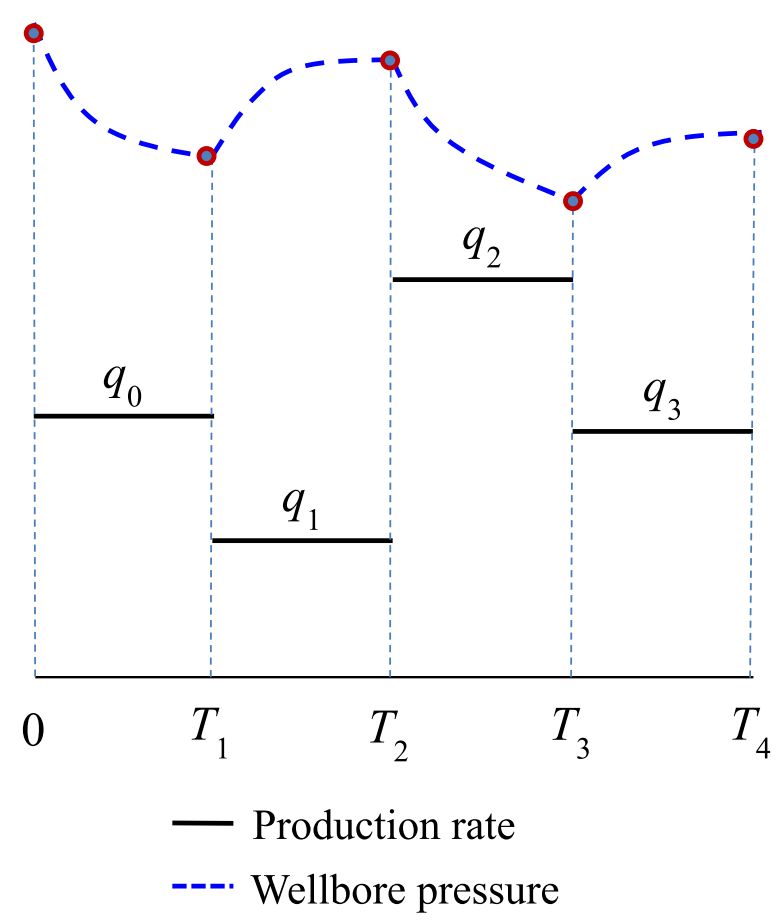

Fig. 1. The production segments with different production rates.

rate functions through adequate piecewise constant production rate steps. In the general cases, the measured production rate data by flowmeters are discrete points, which can be considered as the piecewise constant production rate steps directly. Hence, in our improved deconvolution algorithm, the input data of the variable production rate are piecewise constant production rates.

Here, Laplace transform is not involved in the improved Ilk et al.'s deconvolution algorithm. Based on the above deduced analytical formula from the convolution equation, the deconvolution process based on B-splines can be kept in the level of integral in the real time space as follows.

By using the measured wellbore pressure and variable production rate data for the deconvolution, from Eq. (7) and the analytical solutions of the integration i.e. Eqs. (8)-(10), the over-determined linear system is obtained as follows (Ilk, 2005; Ilk et al., 2005):

$\mathbf{X} \cdot \mathbf{C}=\Delta \mathbf{P}$

where $\mathbf{X}$ is the $m n \times u$ sensitivity matrix; $\mathbf{C}$ is the $u$-vector of undetermined coefficients $c_{i}$; and $\Delta \mathbf{P}$ is the $m n$-vector of measured wellbore pressure drop $p_{i n i}-p_{j k}$; where $m n=\sum_{j=0}^{m-1} n_{j}$. The elements of the sensitivity matrix are as follows:

$\mathbf{X}_{j k, i}=\int_{0}^{T_{k}^{j}} B_{i}^{2}\left(T_{k}^{j}-\tau\right) q(\tau) \mathrm{d} \tau$

In Eq. (12), the value of the integral $\mathbf{X}_{j k, i}$ can be obtained by substituting $t=T_{k}^{j}$ into the analytical formula Eq. (8). It is worth to mention that in order to further improve the deconvolution computation speed, the binary search method is also applied to find which production segment (with constant production rate) the measured wellbore pressure data point $\left(T_{k}^{j}, p_{k}^{j}\right)$ locates at. It is necessary especially for the cases when the quantity of production segments (the number of measured production rate data in reality) is abundant.

In comparison with Ilk et al.'s deconvolution algorithm by using Laplace transformation, the proposed direct analytical solution method of the integration for calculating the elements of the sensitivity matrix can not only guarantee the success of the deconvolution computation process based on B-splines, but also can largely enhance the deconvolution computation speed.

When the level of data error increases, the least square method can't provide a sufficient regularization (Ilk, 2005; Ilk et al., 2005). Therefore, additional regularization is required in order to ensure the relevance of the spline representation with well testing typical curves from reservoir modeling. As in Ilk et al.'s deconvolution algorithm (Ilk, 2005; Ilk et al., 2005), for the over-determined linear system, the following two conditions are appended for each spline interval:

$$
\begin{aligned}
& \alpha\left[\left(t \sum_{i=1}^{u} c_{i} B_{i}^{2}(t)\right)_{t=t_{k}}-\left(t \sum_{i=1}^{u} c_{i} B_{i}^{2}(t)\right)_{t=t_{k+1 / 2}}\right]=0 \\
& \alpha\left[\left(t \sum_{i=1}^{u} c_{i} B_{i}^{2}(t)\right)_{t=t_{k+1 / 2}}-\left(t \sum_{i=1}^{u} c_{i} B_{i}^{2}(t)\right)_{t=t_{k+1}}\right]=0
\end{aligned}
$$

In other words, it is required that the logarithmic derivative of the wellbore pressure corresponding to the constant unit production rate differ slightly between the knot and the middle location between knots (Ilk, 2005; Ilk et al., 2005). When the measured wellbore pressure and production rate have no errors, the regularization parameter $\alpha$ is equal to zero. When the measured data have errors, a positive value of $\alpha$ is selected to eliminate the error effect. The range for the value of $\alpha$ is $[0,1]$.

Then the over-determined linear system which incorporates the appended regularization can be written as follows:

$\alpha \mathbf{X}_{r} \mathbf{C}=0$

$(1-\alpha) \cdot \mathbf{X C}=(1-\alpha) \cdot \Delta \mathbf{P}$

where $\mathbf{X}_{r}$ is the $k n \times u$ matrix; $k n=2 \cdot\left(\sum_{j=0}^{m-1} n_{j}-1\right)$. The linear systems of Eqs. (15) and (16) can be solved together by the least square method (Burden and Faires, 2010) to determine the value of C. Eventually, according to Eq. (5), the derivative of wellbore pressure function $p_{u}^{\prime}$ corresponding to the constant unit production rate can be reconstructed. The log-log typical curves for well testing also need the wellbore pressure $p_{u}$, which can be deduced by the integration of both sides of Eq. (5), as follows:

$\int_{0}^{t} p_{u}^{\prime}(t) \mathrm{d} t=\sum_{i=1}^{u} c_{i} \int_{0}^{t} B_{i}^{2}(t) \mathrm{d} t$

Eq. (17) can be further deduced as follows:

$p_{u}(t)-p_{\text {ini }}=\sum_{i=1}^{u} c_{i} \int_{0}^{t} B_{i}^{2}(t) \mathrm{d} t$

Eq. (18) can be rewritten as follows:

$p_{u}(t)=p_{\text {ini }}+\sum_{i=1}^{u} c_{i} \int_{0}^{t} B_{i}^{2}(t) \mathrm{d} t$

Once the value of $\mathbf{C}$ is determined, the functions of transient wellbore pressure $p_{u}$ and its derivative $p_{u}^{\prime}$ can be obtained by Eqs. (19) and (5), respectively; the data of transient wellbore pressure $p_{\boldsymbol{u}}$ and its derivative $p_{u}^{\prime}$ corresponding to the constant unit production rate can be output for well test analysis. In addition, the wellbore pressure $p$ corresponding to the variable production rate as the reconstructed pressure response by B-splines from Eq. (6) can be compared with the measured wellbore pressure data. It can be used as the constraint for restricting the value assignment of the base $b$ and the regularization parameter $\alpha$ during the regularization process.

\section{Validation of the improved deconvolution algorithm}

The problem considered here involves the radial Darcy's flow in an infinite dual-porosity reservoir. The reservoir is homogeneous, isotropic and isothermal. And the single-phase horizontal flow does not have any gravity effect. Both the wellbore storage and skin effect are considered. The Newtonian fluid and rocks are slightly compressible. 
Table 1

Reservoir parameters.

\begin{tabular}{ll}
\hline Reservoir parameters & Values \\
\hline Wellbore storage coefficient & $0.001 \mathrm{bbl} / \mathrm{psi}$ \\
Skin factor & 5.0 \\
Permeability & $1.0 \mathrm{md}$ \\
Reservoir thickness & $10.0 \mathrm{ft}$ \\
Initial pressure & $50.0 \mathrm{MPa}$ \\
Porosity & 0.1 \\
Well radius & $0.3 \mathrm{ft}$ \\
Viscosity & $1.0 \mathrm{cp}$ \\
Formation volume factor & $1.0 \mathrm{~B} / \mathrm{STB}^{-6} \mathrm{psi}^{-1}$ \\
Total compressibility & $3.0 \times 10^{-1}$ \\
Elastic storage ratio of fracture & 0.1 \\
Inter-porosity flow coefficient & $1.0 \times 10^{-6}$ \\
\hline
\end{tabular}

Table 2

The data of production rate.

\begin{tabular}{ll}
\hline Production duration (Day) & Production rate (STB/D) \\
\hline 1 & 0.5 \\
4 & 1.0 \\
5 & 0.75 \\
10 & 1.25 \\
30 & 2.25 \\
50 & 1.0 \\
100 & 1.5 \\
200 & 1.0
\end{tabular}

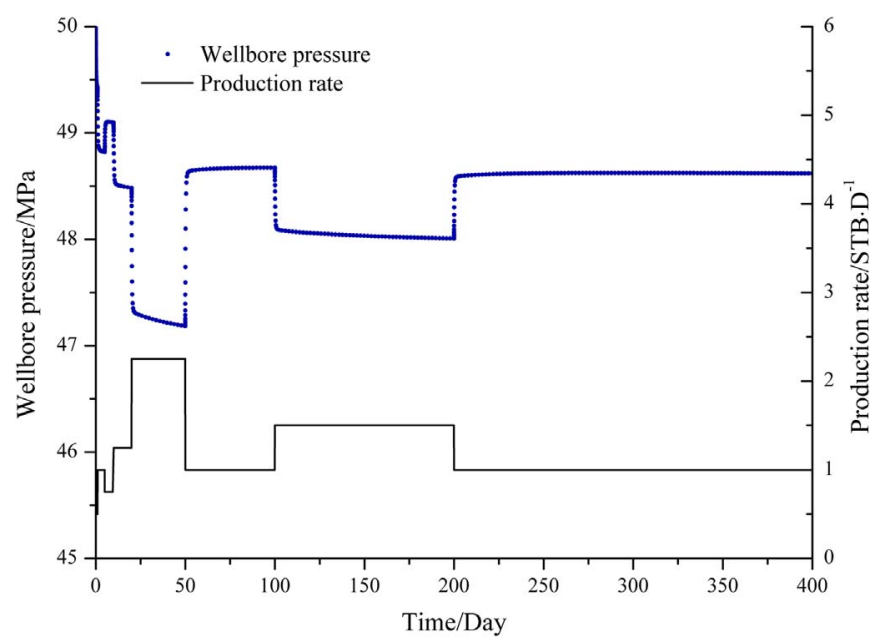

Fig. 2. Wellbore pressure response corresponding to the variable production rate.

The values of reservoir parameters are shown in Table 1. The production history for a production well includes eight production durations with different production rates (see Table 2 for details). The data for the wellbore pressure response corresponding to the variable production rate is shown in Fig. 2. The total number of wellbore pressure data is 426 . The initial pressure is $50.0 \mathrm{MPa}$. The exact solution of the wellbore pressure response corresponding to the constant unit production rate 1.0 STB/D for 400 days is shown in Fig. 3.

Then the improved deconvolution algorithm can be adopted to transfer the wellbore pressure response corresponding to the discontinuous variable production rate to the one corresponding to the constant unit production rate. Here, the value of $b$ is set as 1.8; the regularization parameter $\alpha$ is set as 0 . The aforementioned exact solution can be used to verify its correctness.

Fig. 4 shows the comparison of the deconvolution results regarding the wellbore pressure drop and its derivative by our deconvolution algorithm with the exact solutions. From Fig. 4, it can be seen that the

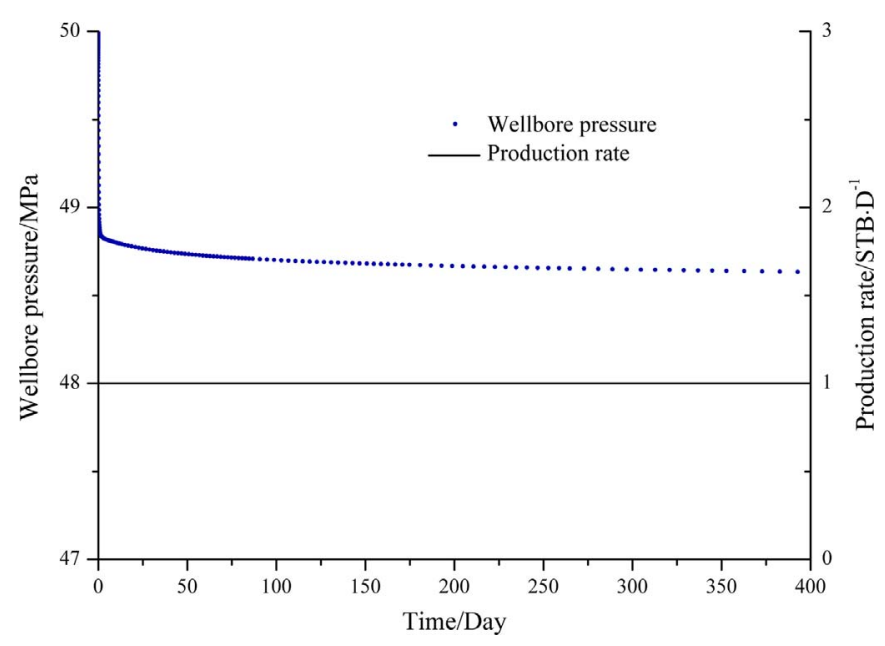

Fig. 3. Wellbore pressure response corresponding to the constant unit production rate.

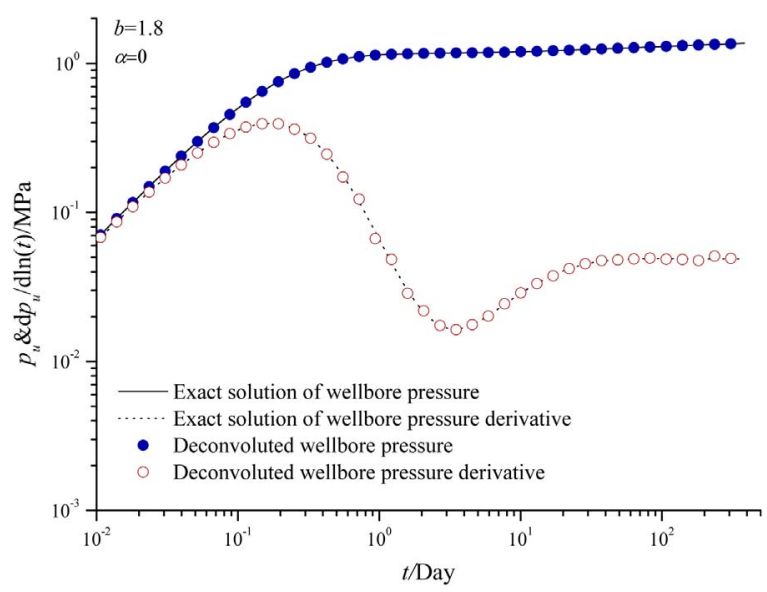

Fig. 4. Comparison of the deconvolution results with the exact solutions.

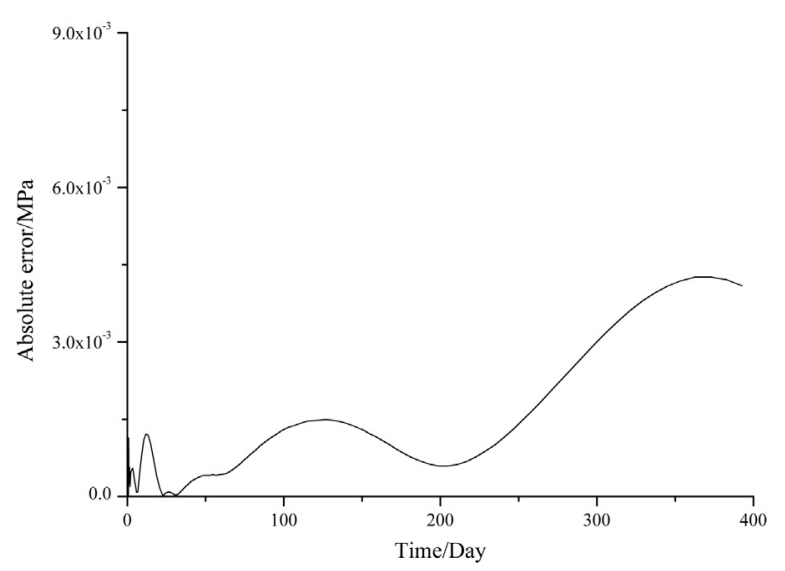

Fig. 5. The absolute error curve.

deconvolution results have good agreement with the exact solutions. Figs. 5 and 6 show the absolute error and relative error for the deconvolved wellbore pressure, respectively; it can be seen that the absolute and relative errors both tend to increase with the time increasing; the absolute error is less than $0.006 \mathrm{MPa}$, and the relative error is no more than $0.4 \%$, which indicates high accuracy of the improved deconvolution algorithm. Besides, the computation time for the improved deconvolution process is just $0.01 \mathrm{~s}$ by using an ordinary computer, which is equipped with double central processing units of $3.40 \mathrm{GHz}$ core frequency and random access memory of $4.00 \mathrm{~GB}$ size. The improved deconvolution algorithm shows high computation speed. 


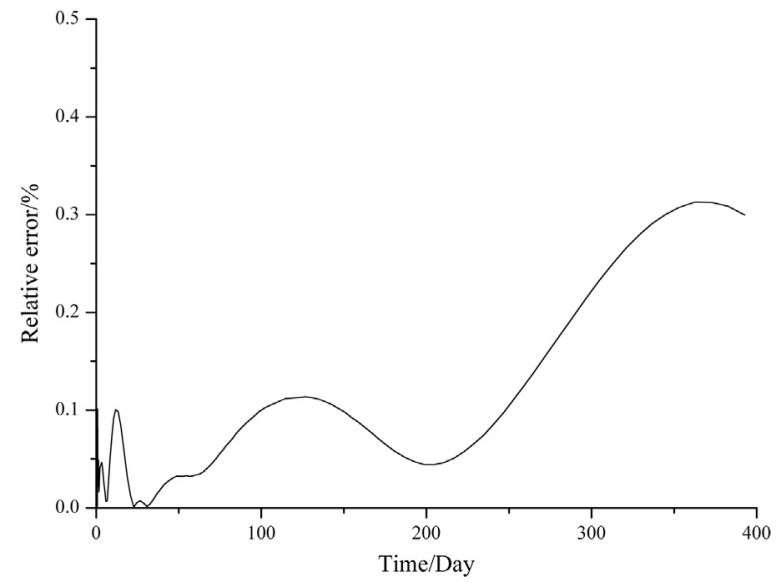

Fig. 6. The relative error curve.

\section{Stability test of the improved deconvolution algorithm}

\subsection{Case study for stability test when data error exists}

In order to test the stability of the improved deconvolution algorithm, a 5\% random relative error is added into the measured wellbore pressure drop corresponding to the same variable production rate data as in Section 3, which is shown in Fig. 7. Due to the existence of data errors, the aforementioned regularization method has to be employed to overcome the effect of data errors in the deconvolution process. It has been recommended that the selection of the optimum values of the regularization parameter $\alpha$ and the base $b$ for logarithmic distribution of B-spline knots should be combined together in order to efficiently eliminate the data noise (Ilk, 2005; Ilk et al., 2005). As the constraint of the value assignment of the two parameters, it should be guaranteed that the reconstructed wellbore pressure response by Bsplines can match the measured wellbore pressure data.

In order to smooth the typical curves, the value of the regularization parameter $\alpha$ is set as the largest one under the constraint. Then the value selection of the base $b$ is the key job. According to Ilk et al.'s suggestion that the number of knots should be on the order of at least 2-6 knots per log cycle (Ilk, 2005; Ilk et al., 2005), it can be deduced from Eq. (2) that the value of the base $b$ should be on the order of 1.473.16. Fig. 8 shows the comparison of the deconvolution results under different values of the base $b$ from the data with errors with the exact solutions. From Fig. 8(A), it can be seen that if the value of $b$ is too small, the regularization is insufficient; the distribution of the wellbore

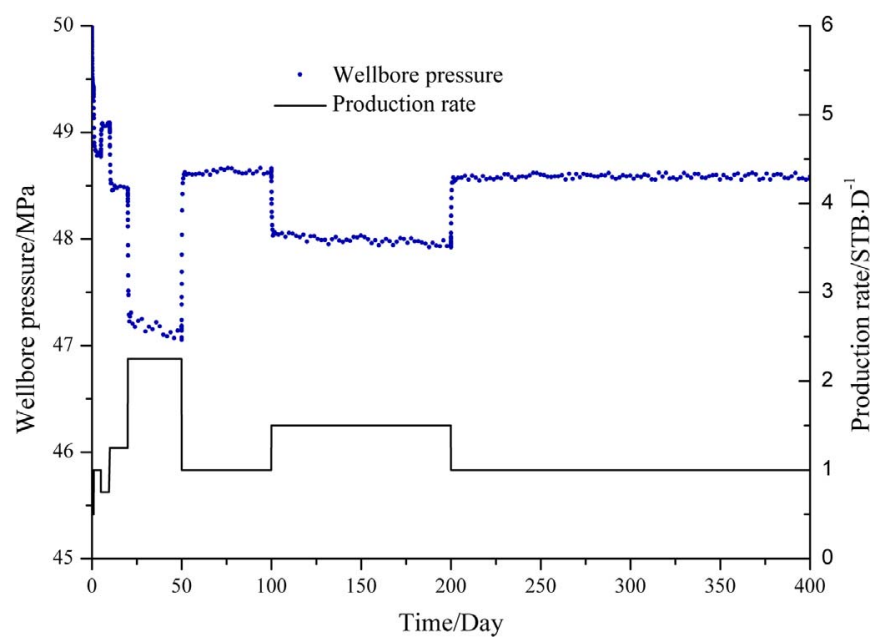

Fig. 7. Wellbore pressure drop with $5 \%$ random relative error corresponding to the variable production rate. pressure derivative $p_{u}^{\prime}$ points from the deconvolution results is dispersive, and out of order. Then it will lead to the difficulty in the well test analysis for the deconvolved wellbore pressure data due to its disorder and uncertainty. If the value of $b$ is too large, the distribution of the wellbore pressure derivative points becomes too tight, and sharp turning emerges; some basic reservoir-model characteristics of typical log-log curves of wellbore pressure derivative for identifying a relevant reservoir model during the well test analysis process can be removed, as shown in Fig. 8(C).

From Fig. 8(B), it can be noted that by tuning the value of regularization parameter $\alpha$ as 0.01 and setting the value of $b$ as 2.6, the deconvolution algorithm can successfully recover the transient wellbore pressure features of the reservoir model for the whole production duration. And for the constraint, the reconstructed wellbore pressure response by B-splines still matches the measured wellbore pressure data (see Fig. 9). The improved Ilk et al.'s deconvolution algorithm exhibits its good stability of data error tolerance.

From the deconvolution results, it can also be concluded that the deconvolved wellbore pressure $p_{\boldsymbol{u}}$ has better agreement with the related exact solutions than the deconvolved wellbore pressure derivative $p_{u}^{\prime}$. The reason is that the deconvolved wellbore pressure is more tolerant to the data errors than the devonvolved wellbore pressure derivative.

\subsection{Statement on how to perform the regularization}

From the above case study for testing the stability of the improved deconvolution algorithm, it can be seen that determination of coefficients $b$ and $\alpha$ is very important in the regularization process when data errors exist, because different coefficient value assignment may lead to different deconvolution results.

Through many numerical experiments for the improved deconvolution algorithm, it is found that when the initial formation pressure is known, the deconvolved wellbore pressure $p_{u}$ is unique and very stable, and tuning the coefficients $b$ and $\alpha$ have little effect on the typical loglog curves of the deconvolved wellbore pressure; the key problem is that the typical log-log curves of the deconvolved wellbore pressure derivative $p_{u}^{\prime}$ is very sensitive to the data errors (The same problem also exists in other deconvolution algorithms such as von Schroeter et al.'s algorithm and Levitan et al.'s algorithm.), and the regularization is performed mainly for smoothing the typical log-log curves of the deconvolved wellbore pressure derivative by tuning the coefficients $b$ and $\alpha$, so as to reduce the uncertainty of the subsequent well testing interpretation by the typical curve analysis method. However, it is fortunate that the measured data for well testing generally have high accuracy (in comparison with the wellbore production data). Therefore, the deconvolution approach can still be widely applied in well test analysis. For the improved deconvolution algorithm, it is found that as the value assignment of $\alpha$ satisfies the constraint, and the value of the base $b$ is set on the order of $1.47-3.16$, the basic reservoir-model characteristics of typical log-log curves of the wellbore pressure drop and the wellbore pressure derivative can be identified from the deconvolution results through the regularization process.

In order to deconvolve a correct wellbore pressure derivative $p_{u}^{\prime}$ for the well testing by the deconvolution approach and the involved regularization method, it is also necessary to make full use of an accurate knowledge of initial pressure, the knowledge from the sources of reservoir geology and reservoir characterization (such as a pressure build up testing), and some engineering experiences (Osman and Thwaites, 2014). For example, if it is known from the sources of reservoir geology that the reservoir is a dual-porosity reservoir, it can provide the information that the typical log-log curves of the deconvolved wellbore pressure derivative should have the seepage flow features in dual-porosity reservoirs. And if a pressure build up testing can be conducted at a shut-in period in advance, its accurate typical $\log$-log curves of the wellbore pressure derivative in the relatively short 
A

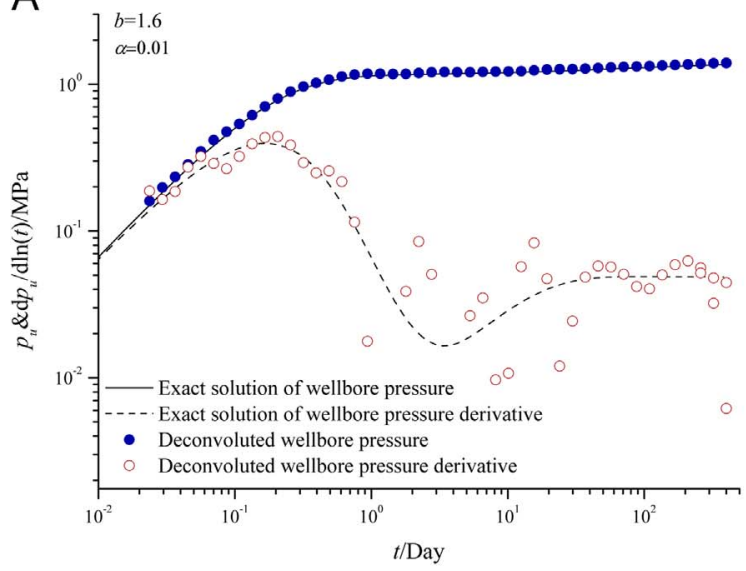

B

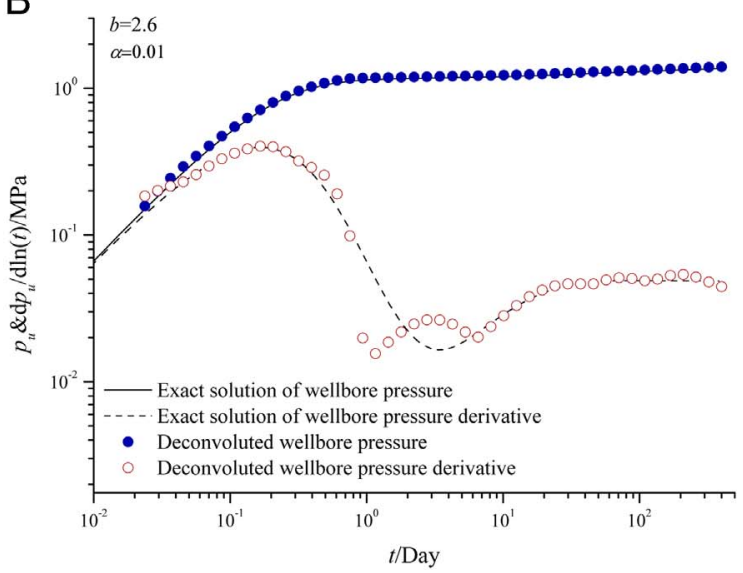

C

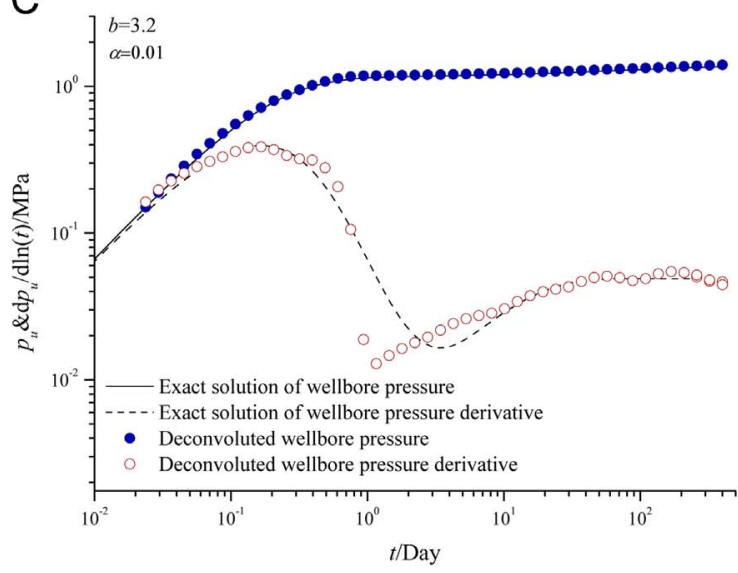

Fig. 8. Comparison of the deconvolution results with the exact solutions.

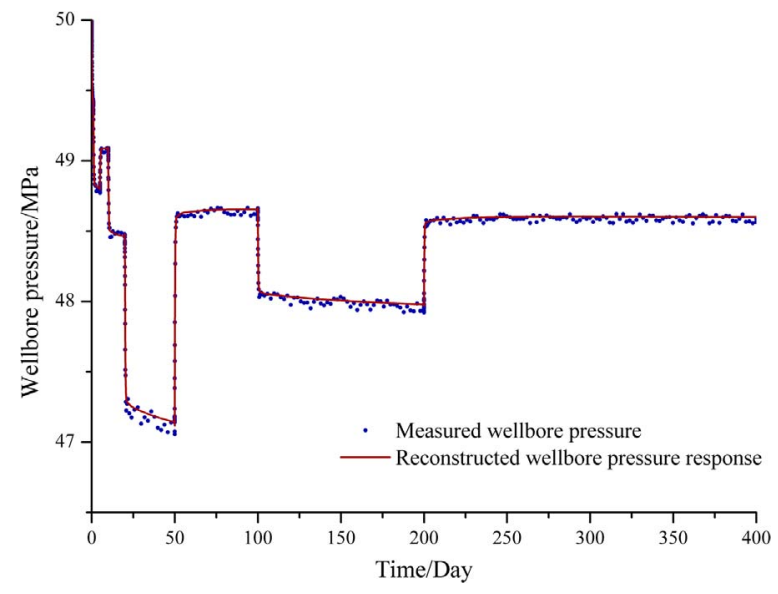

Fig. 9. Comparison of the reconstructed wellbore pressure response with the measured wellbore pressure data.

period can give the direct guidance for deconvolving the wellbore pressure derivative at the whole period; after all, they belong to the same typical curves for reservoir model identification, just with different time length.

\section{Application to field example}

Here, the improved deconvolution algorithm is applied to a real field example of the well SapGS02 from the example file of the KAPPA software. The production history for a production well includes thirteen production periods with different piecewise constant production rates,

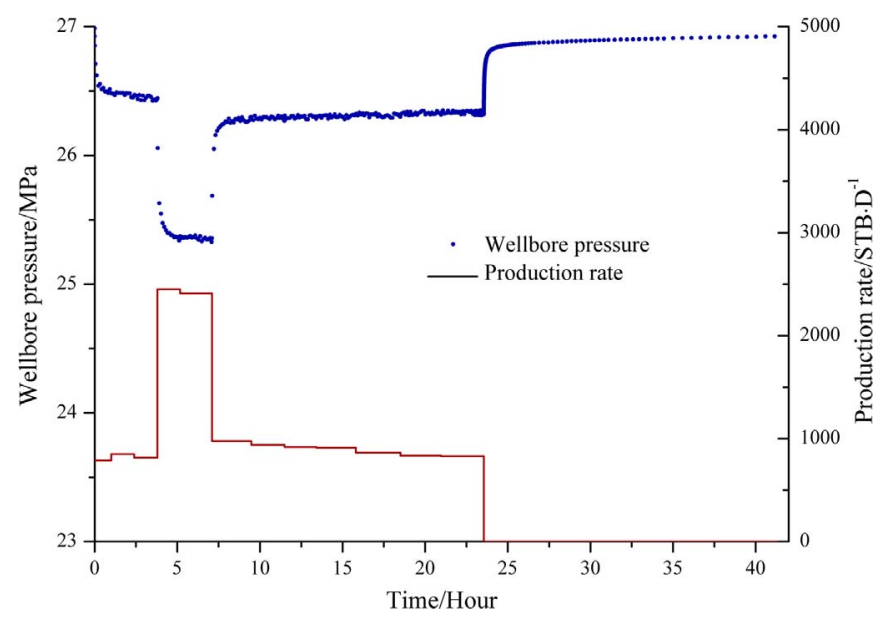

Fig. 10. The data of the wellbore pressure and the corresponding production rate for the well SapGS02.

among which the last one is the well shut-in period; the corresponding wellbore pressure is measured. The data of the production rate and the wellbore pressure are shown in Fig. 10. The total number of the data points for the measured wellbore pressure is 485 . The initial pressure is 27.0 MPa.

The improved deconvolution algorithm is applied in order to transfer the wellbore pressure data corresponding to the discontinuous variable production rate into the one corresponding to the constant unit production rate for the entire production history. The computation time is just $0.015 \mathrm{~s}$. Fig. 11 shows the typical log-log curves of the 


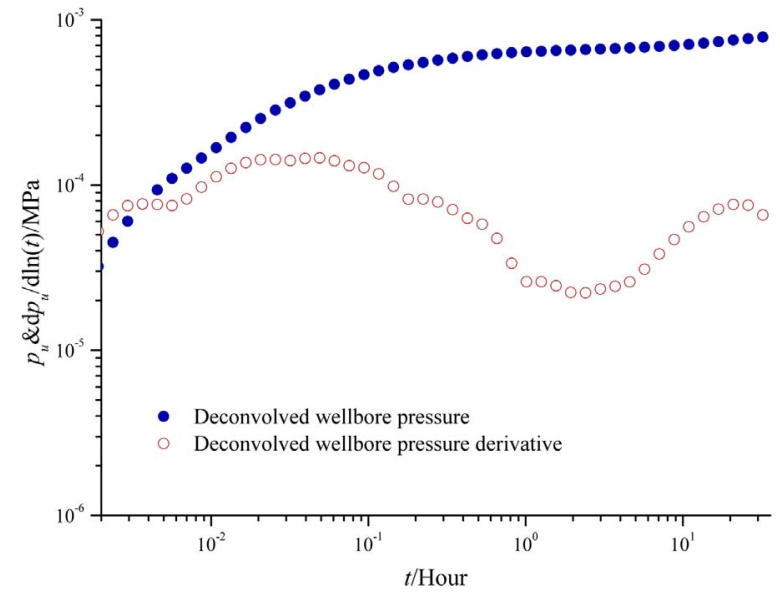

Fig. 11. The typical log-log curves of the wellbore pressure drop and the wellbore pressure derivative from the deconvolution results.

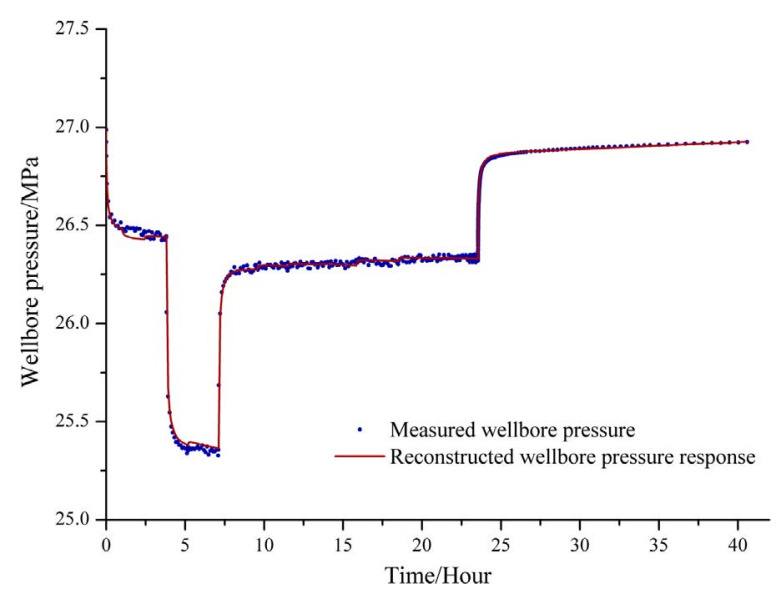

Fig. 12. Comparison of the reconstructed wellbore pressure response with the measured wellbore pressure data.

wellbore pressure drop and the wellbore pressure derivative from the deconvolution results; where the value of regularization parameter $\alpha$ is set as 0.003 and the value of $b$ is set as 2.3. The reconstructed wellbore pressure response by B-splines matches the measured wellbore pressure data very well in Fig. 12.

The typical log-log curves calculated from the wellbore pressure data in the last well shut-in period are also plotted, as shown in Fig. 13. From Figs. 12 and 13, it can be seen that the deconvolved typical log-

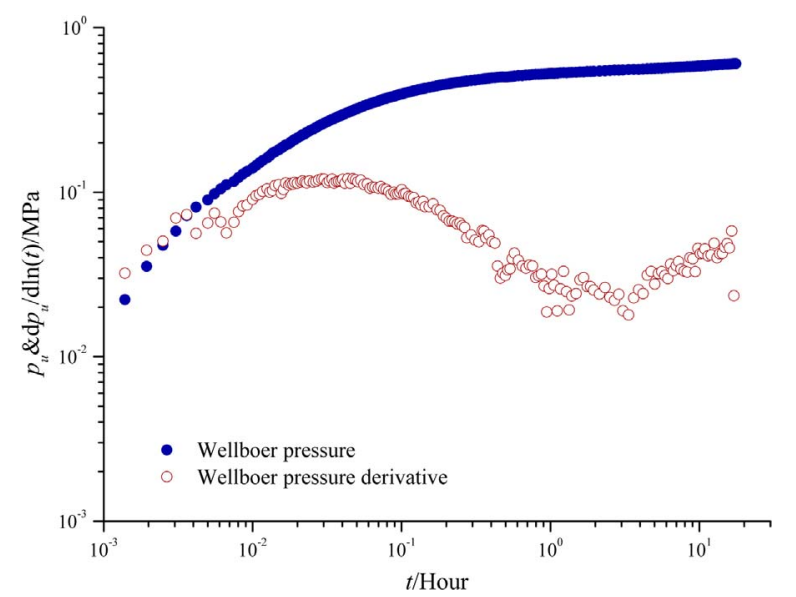

Fig. 13. The typical log-log curves calculated from the wellbore pressure data in the last well shut-in period.

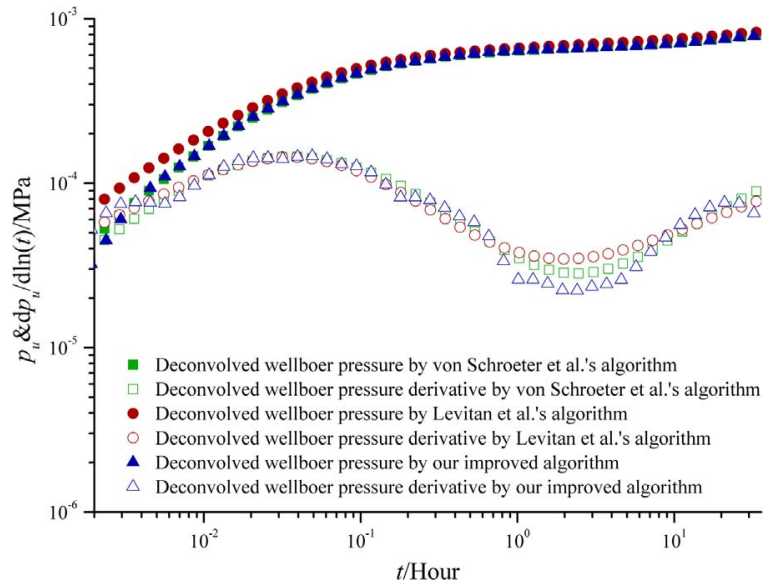

Fig. 14. Comparison of three couples of typical log-log curves of the wellbore pressure drop and the wellbore pressure derivative by three different deconvolution algorithms.

log curves have very similar features with the ones directly from the data in the well shut-in period.

Besides, the deconvolution results by von Schroeter et al.'s algorithm and Levitan et al.'s algorithm are also obtained through the KAPPA Software, respectively; wherein the regularization parameters for the two algorithms are both set as the default values in the Software. Fig. 14 shows the comparison of three couples of typical log-log curves of the wellbore pressure drop and the wellbore pressure derivative, which are plotted from the deconvolved results by the three different deconvolution algorithms, respectively. From Fig. 14, it can be seen that the three couples of log-log typical curves have good agreement, as a whole. The two wellbore pressure drop curves, obtained by von Schroeter et al.'s deconvolution algorithm and our improved deconvolution algorithm respectively, are very close; however, they both have some difference from the wellbore pressure drop curve obtained by the Levitan et al.'s deconvolution algorithm at the early stage. The wellbore pressure derivative curves obtained by the three deconvolution algorithms also have some difference at the "concave" parts; however, the difference could have little effect on the well testing interpretation for the deconvolved data. From Figs. 14 and 13, it is also indicated that the feature of the typical log-log curves corresponding to the improved deconvolution algorithm is very close to the one of the typical log-log curves calculated directly from the wellbore pressure data in the last well shut-in period.

In addition, through many numerical experimental tests, it is also concluded that as the quantity of data largely increases, the improved Ilk et al.'s deconvolution algorithm exhibits the big advantage in fast computational speed over von Schroeter et al.'s algorithm and Levitan et al.'s algorithm.

\section{Conclusions}

It is realized that the variable production rate commonly have large discontinuities in reality, and then Duhamel principle can't be transformed by Laplace transformation. It is the root of the two aforementioned problems existent in Ilk et al.'s deconvolution algorithm. Here, in terms of the root of the problems, Ilk et al.'s deconvolution algorithm is improved fundamentally: We keep the computation process of the deconvolution in the level of integral in the real time space instead of the Laplace space because it has lower requirement for the continuity of the variable production rate. According to the real production rate history, a technique of piecewise analytical integration is adopted for obtaining the elements of sensitivity matrix in the deconvolution process. The proposed analytical integration method can not only largely enhance the computation speed in comparison with the timeconsuming numerical integration method, but also guarantee the 
success of the deconvolution computation process due to the reason that Laplace transformation can be avoided. Besides, as in Ilk et al.'s deconvolution algorithm, the same regularization method is incorporated for eliminating the effect of data error.

Numerical experimental tests demonstrate that the improved deconvolution algorithm exhibits good accuracy, computation speed and stability of data error tolerance. And the statement on how to perform the regularization when data error exists is also made in order to deconvolve the correct wellbore pressure derivative. Through the application of the improved algorithm into a field example, it is indicated that by and large the deconvolution results by the improved algorithm have good agreement with the ones by von Schroeter et al.'s algorithm and Levitan et al.'s algorithm; and the feature of typical log$\log$ curves of the wellbore pressure drop and the wellbore pressure derivative corresponding to the improved algorithm is very close to the one of typical log-log curves calculated directly from the wellbore pressure data in the last well shut-in period.

What's more, as the quantity of data largely increases, the improved Ilk et al.'s algorithm exhibits the big advantage in the fast computational speed over von Schroeter et al.'s algorithm and Levitan et al.'s algorithm. As a result, the improved Ilk et al.'s deconvolution algorithm is more suitable for dealing with the deconvolution problems with large amounts of data.

\section{Acknowledgement}

The authors would like to acknowledge the fundings by the project (Grant no. 51404232) sponsored by the Natural Science Foundation of China (NSFC), the National Science and Technology Major Projects (Grant no. 2011ZX05038003; Grant no. 2011ZX05046-03) and the project (Grant no. 2014M561074) by the China Postdoctoral Science Foundation.

\section{References}

Burden, R.L., Faires, J.D., 2010. Numerical Analysis 9th ed.. Brooks/Cole, Boston, USA.
Cumming, J.A., Wooff, D.A., Whittle, T., Gringarten, A.C., 2013. Multiwell deconvolution. In: SPE 166458 Presented at the 2013 SPE Annual Technical Conference and Exhibition, New Orleans, USA, 30 September-2 October.

Çınar, M., IlK, D., Onur, M., Valkó, P.P., Blasingame, T.A., 2006. A comparative study of recent robust deconvolution algorithms for well-test and production-data analysis. In: SPE 102575 Presented at the 2006 SPE Annual Technical Conference and Exhibition, San Antonio, Texas, USA, 24-27 September.

Gringarten, A.C., 2010. Practical use of well test deconvolution. In: SPE 134534 Presented at the 2010 SPE Annual Technical Conference and Exhibition, Florence, Italy, 20-22 September.

Ilk, D., 2005. Deconvolution of Variable Rate Reservoir Performance Data Using Bsplines. Texas A\& M University, College Station, Texas, (December 2005).

Ilk, D., Valkó, P.P., Blasingame, T.A., 2005. Deconvolution of variable-rate reservoirperformance data using B-splines. In: SPE 95571 Presented at the 2005 SPE Annual Technical Conference and Exhibition, Dallas, Texas, USA, 9-12 October.

Kim, J., Jang, Y., Ertekin, T., Sung, W.M., 2015. Production analysis of a shale gas reservoir using modified deconvolution method in the presence of sorption phenomena. In: SPE 177320 Presented at the 2015 SPE Eastern Regional Meeting, Morgantown, West Virginia, USA, 13-15 October.

Levitan, M.M., 2005. Practical application of pressure/rate deconvolution to analysis of real well tests. SPE Reserv. Eval. Eng. 8 (2), 113-121.

Levitan, M.M., Crawford, G.E., Hardwick, A., 2006. Practical considerations for pressurerate deconvolution of well-test data. SPE J. 11 (1), 35-47.

Osman, M.S., Thwaites, N., 2014. The application of well test deconvolution to wireline formation tester pressure buildup and falloff data to improve coalbed methane reservoir characterization. In: SPE 167764 Presented at the 2014 SPE/SAGE European Unconventional Conference and Exhibition, Vienna, Austria, 25-27 February.

Onur, M., Çınar, M., IlK, D., Valkó, P.P., Blasingame, T.A., Hegeman, P.S., 2006. An investigation of recent deconvolution methods for well-test data analysis. In: SPE 102575 Presented at the 2006 SPE Annual Technical Conference and Exhibition, San Antonio, Texas, USA, 24-27 September.

Pimonov, E., Ayan, C., Onur, M., Kuchuk, F., 2009. A new pressure/rate-deconvolution algorithm to analyze wireline-formation-tester and well-test data. In: SPE 123982 Presented at the 2009 SPE Annual Technical Conference and Exhibition, New Orleans, USA, 4-7 October.

Vaferi, B., Eslamloueyan, R., 2015. Hydrocarbon reservoirs characterization by cointerpretation of pressure and flow rate data of the multi-rate well testing. J. Pet. Sci. Eng. 135 (2015), 59-72.

von Schroeter, T., Hollaender, F., Gringarten, A.C., 2002. Analysis of well test data from downhole permanent downhole gauges by deconvolution. In: SPE 77688 Presented at the 2002 SPE Annual Technical Conference and Exhibition, San Antonio, Texas, USA, 29 September-2 October.

von Schroeter, T., Hollaender, F., Gringarten, A.C., 2004. Deconvolution of well test data as a nonlinear total least squares problem. SPE J. 9 (4), 375-390. 